\title{
Color y sexo
}

\section{Color and sex}

\author{
Dominga González *, Encarnación Sueiro** \\ * Universidad de Vigo, **C.O.F. Nóvoa Santos-SERGAS-Ourense
}

\begin{abstract}
Resumen
La población infantil puede ser influenciada por los estereotipos de género que hay sobre los colores. En relación a este hecho, se llevó a cabo un estudio en el que participaron 50 niños/as de entre 4 y 7 años de edad. El objetivo principal era investigar cómo influye el sexo y la información relacionada con él en el uso y la elección de los colores que hacen ellos/as. Los resultados obtenidos reflejan que los colores utilizados son diferentes dependiendo del sexo de la ilustración y que existen diferencias entre los colores favoritos de los niños y de las niñas.

Palabras clave: infancia, color, sexo, género, estereotipos.
\end{abstract}

\begin{abstract}
Children can be influenced by gender stereotypes about colors. In relation to this fact, a study was carried out involving 50 children between 4 and 7 years of age. The main objective was to investigate how gender and information relation to it, influence in color's use and choice by children. The results obtained reflect that the colors used are different depending on the sex of the illustration and there are differences between the favourite colors of boys and the favourite colors of girls. Keywords: childhood, color, gender, stereotypes.
\end{abstract}

\section{Introducción}

Dado que la población infantil es la más vulnerable a todo tipo de influencias y también la que tiene más contacto con el mundo de los colores, sería interesante estudiar el grado de extensión que tiene, hoy en día, el prejuicio referente al color que separa los colores de las niñas y de los niños. Por esta razón se llevó a cabo el estudio que se explica a continuación, con el objetivo de conocer la influencia que ejerce el sexo sobre la elección de los colores y su uso, por parte de las niñas y de los niñosEn la investigación participaron alumnas y alumnos de $5^{\circ}$ Educación Infantil (4-5 años) y $1^{\circ}$ Educación Primaria (6-7 años). El motivo de la elección de estas edades se debe a que el primer intervalo (4-5 años) se corresponde con el inicio de una fase de interiorización sexual, en la que van aumentando los conocimientos sobre los estereotipos y los roles de género; y el otro intervalo (6-7 años) representa el final de esta misma fase. Por lo tanto, se pueden comparar los resultados obtenidos en ambos intervalos de edades, observando si existen similitudes o diferencias.

Conocer la información que se va a presentar a continuación resulta de gran utilidad para combatir los estereotipos de género en las aulas, donde se trabaja con los colores, especialmente en las primeras etapas educativas.

\section{Objetivos}

El objetivo principal era conocer la existencia de diferencias en las preferencias de color y su uso, por parte de las niñas y de los niños con edades comprendidas entre los 4 y los 7 años.

\section{Hipótesis}

A continuación se presentan las hipótesis que fueron formuladas para el estudio realizado.

\section{Hipótesis en función del sexo}

Elección de la portada del cuaderno. H1: En cuanto a la elección de las portadas de los cuadernos se pronostican diferencias entre las niñas y los niños.

Elección de la figura de la niña o del niño para pintar. H2: Quienes participan en las pruebas, elegirán para colorear, en mayor medida, la figura que es de su mismo sexo.

Variedad de colores utilizados para colorear las figuras de la niña y del niño. H3: Las niñas y los niños utilizarán mayor número de colores para pintar la figura que es de su mismo sexo que para colorear la que es del sexo contrario.

Barrera de género en los colores empleados para niñas y niños a la hora de pintar las dos figuras sexuadas. H4: A la hora de colorear las figuras de la niña y del niño habrá diferencias entre los colores empleados para cada tipo de figura.

Dibujo de las estrellas. H5: Habrá bastante coincidencia en los colores que utilicen las niñas y los niños para pintar las tres estrellas.

Color favorito. H6a: Los niños indicarán, mayoritariamente, el azul como su color favorito. H6b: Las niñas indicarán, mayoritariamente, el rosa como su color favorito. 


\section{Hipótesis en función de la edad}

Diferencias en el uso de los colores, según los grupos de edad. $H 7$ : A la hora de pintar las figuras de la niña y del niño, habrá más diferencias en la elección de los colores que hace el alumnado de 6-7 años que el de 4-5 años.

\section{Otras hipótesis}

Presencia del color favorito en una de las estrellas pintadas. H8: Las y los participantes pintarán de su color favorito una de las tres estrellas presentadas.

\section{Método}

\section{Participantes}

En el estudio participaron un total de 50 alumnos/as de un colegio de la ciudad de Ourense (Galicia), pertenecientes a los cursos de $5^{\circ}$ Educación Infantil (45 años) y $1^{\circ}$ Educación Primaria (6-7 años). En la muestra total de participantes había 22 niñas y 28 niños.

\section{Instrumentos}

Para la recogida de datos se utilizaron dos tipos de cuadernos para colorear, cuyas portadas eran diferentes, y el cuestionario de observación de la actitud de cada participante ante la tarea de colorear. Ambos instrumentos fueron de elaboración propia y los supervisaron ocho jueces externos.

\section{Procedimiento}

Las condiciones en las que se desarrolló la tarea de colorear fueron siempre las mismas, para tratar, en la medida de lo posible, que los resultados no se viesen alterados, La prueba se pasó a cada participante de forma individual y no hubo límite de tiempo.

A cada sujeto se le dio a elegir entre dos cuadernos de portadas diferentes, pero con el mismo contenido de figuras para colorear en su interior. Una de las portadas tenía la imagen del coche de la película de Cars sobre un fondo rosa, mientras que la otra presentaba la imagen de Elsa, la protagonista de Frozen sobre un fondo azul. Una vez escogido el cuaderno por su portada, tuvieron que elegir entre pintar la figura de la niña o del niño. Cabe mencionar que las figuras de la niña y del niño fueron presentados de forma aleatoria en el interior de los cuadernos, de forma que en algunos aparecía la figura de la niña inmediatamente después de la portada y en otros, era la figura del niño la que aparecía primero. La idea de pedir a cada participante que escogiese entre pintar la niña o del niño se propuso con la intención de averiguar por cuál de las dos figuras se inclinaban más. Luego de pintar la pintura escogida se les pidió que marcasen con cruces de los colores que emplearían para pintar la otra figura que no habían seleccionado. A continuación tuvieron que colorear las tres estrellas que aparecían en la última hoja del cuaderno, con la única condición de que cada estrella debía ser pintada de un color diferente. Para la tarea de colorear se les ofrecieron diez pinturas de los colores rosa, rojo, lila, violeta, verde claro/oscuro, azul claro/oscuro, amarillo y naranja.
Los colores que emplearon para cada figura fueron anotados en un cuestionario de respuesta cerrada, al igual que su sexo y edad y la elección que hicieron de las portadas y de la figura a pintar (niña o niño). Además, en el cuestionario había una última pregunta que se les hizo al final de la prueba donde se les pedía que señalasen su color preferido de los diez ofrecidos para la realización de la tarea de colorear.

\section{Análisis de datos}

Los datos obtenidos en los cuestionarios de las pruebas fueron introducidos en el programa de estadística PSPP, para crear, seguidamente las tablas necesarias para el análisis y la verificación de las hipótesis formuladas. Pero debido a que la muestra de estudio era pequeña, no se pudieron hallar diferencias significativas en todos los casos planteados por las hipótesis formuladas.

\section{Resultados}

\section{Elección de la portada del cuaderno (H1)}

Como se puede observar en la Figura 1, existen diferencias significativas en la elección de portadas que hacen las niñas y los niños, pues $p$ tiene un valor de 0.00 , es decir, es menor a 0.05 ; y este hecho implica que existan diferencias estadísticamente significativas. En un alto porcentaje $(81,82 \%)$ ellas eligen la portada de Elsa, mientras que el $92,86 \%$ de los niños escogen la portada de Cars.

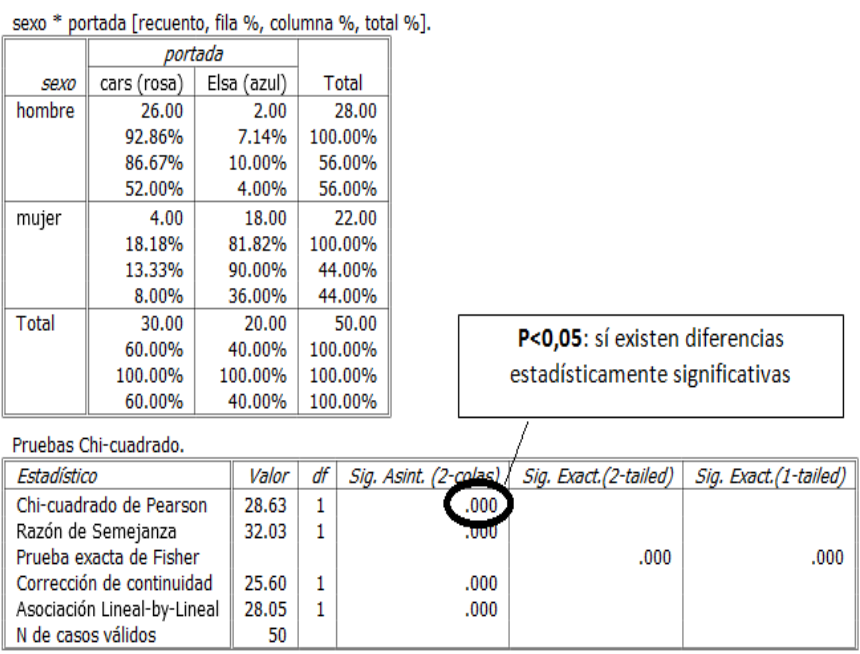

Figura 1. Elección de la portada de los cuadernos. 
Elección de la figura de la niña o del niño para pintar $(\mathrm{H} 2)$

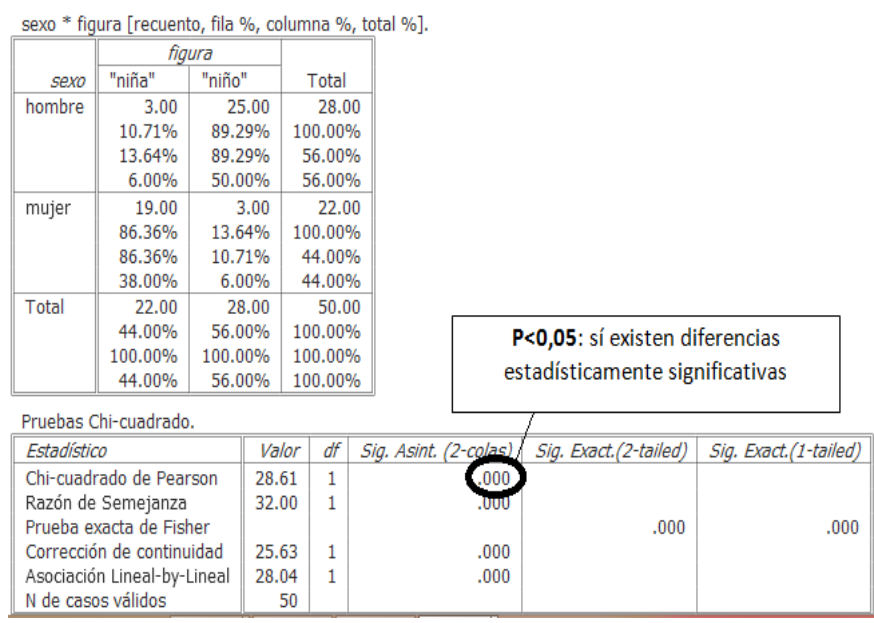

Figura 2. Elección de la figura de la niña o del niño para pintar.

También se puede ver en la Figura 2 que existen diferencias estadísticamente significativas en la elección de la figura a pintar por parte de los niños y de las niñas, pues $p$ tiene un valor de 0.00 y es menor que 0.05 . La mayoría de las niñas (un $86,36 \%$ ) escogió para pintar la figura de la niña y un alto porcentaje de ellos (un 89,29\%) eligió la figura del niño.

Variedad de colores utilizados para colorear las figuras de la niña y del niño (H3)

sexo * colores_figura_niño_cat [recuento, fila \%, columna \%, total \%]

\begin{tabular}{||r||r|r|r||}
\hline \multirow{4}{*}{ sexo } & \multicolumn{2}{|c||}{ colores_figura_niño_cat } & \multicolumn{1}{c||}{} \\
\cline { 2 - 4 } & $3-4$ colores & $5-6$ colores & \multicolumn{1}{c|}{ Total } \\
\hline hombre & 12.00 & 16.00 & 28.00 \\
& $42.86 \%$ & $57.14 \%$ & $100.00 \%$ \\
& $60.00 \%$ & $53.33 \%$ & $56.00 \%$ \\
\hline mujer & $24.00 \%$ & $32.00 \%$ & $56.00 \%$ \\
& 8.00 & 14.00 & 22.00 \\
& $36.36 \%$ & $63.64 \%$ & $100.00 \%$ \\
& $40.00 \%$ & $46.67 \%$ & $44.00 \%$ \\
\hline Total & $16.00 \%$ & $28.00 \%$ & $44.00 \%$ \\
& 20.00 & 30.00 & 50.00 \\
& $40.00 \%$ & $60.00 \%$ & $100.00 \%$ \\
& $100.00 \%$ & $100.00 \%$ & $100.00 \%$ \\
\hline & $40.00 \%$ & $60.00 \%$ & $100.00 \%$ \\
\hline
\end{tabular}

sexo $*$ colores_figuras_niña_cat [recuento, fila \%, columna $\%$, total $\%$ ]

\begin{tabular}{|r||r|r|r||}
\hline \multirow{4}{*}{ sexo } & \multicolumn{1}{||r}{ colores_figuras_niña_cat } & \multicolumn{1}{c||}{} \\
\cline { 2 - 4 } hombre & $3-4$ colores & $5-6$ colores & \multicolumn{1}{c|}{ Total } \\
& 12.00 & 16.00 & 28.00 \\
& $42.86 \%$ & $57.14 \%$ & $100.00 \%$ \\
& $80.00 \%$ & $45.71 \%$ & $56.00 \%$ \\
\hline mujer & $24.00 \%$ & $32.00 \%$ & $56.00 \%$ \\
& 3.00 & 19.00 & 22.00 \\
& $13.64 \%$ & $86.36 \%$ & $100.00 \%$ \\
& $20.00 \%$ & $54.29 \%$ & $44.00 \%$ \\
Total & $6.00 \%$ & $38.00 \%$ & $44.00 \%$ \\
& 15.00 & 35.00 & 50.00 \\
& $30.00 \%$ & $70.00 \%$ & $100.00 \%$ \\
& $100.00 \%$ & $100.00 \%$ & $100.00 \%$ \\
& $30.00 \%$ & $70.00 \%$ & $100.00 \%$ \\
\hline \hline
\end{tabular}

Figura 3. Número de colores utilizados por cada sexo para pintar las figuras del niño (arriba) y de la niña (abajo).
En este caso solo se observan diferencias significativas a la hora de pintar la figura de la niña, donde se obtuvo un valor de $p$ de 0.025 , es decir, menor de 0.05 . Los niños pintaron prácticamente con el mismo número de colores ambos dibujos, como se puede ver en la Figura 3; pero sí que se percibe una diferencia en el caso de las niñas, quienes emplearon más colores para pintar la figura de su mismo sexo.

Barrera de género en los colores empleados por niñas y niños a la hora de pintar las dos figuras sexuadas (H4)

Quienes participaron coinciden en utilizar, especialmente, los colores rosa y lila para la figura de la niña, además de rojo, verde claro y, en un porcentaje menor, azul oscuro. Sin embargo, para la figura del niño, utilizaron, principalmente, el color rojo, seguido de los colores verde claro, azul oscuro y violeta. El lila y el rosa son prácticamente nulos en la figura del niño.

Se puede decir que en general no existen diferencias entre los colores que utilizan ellas y los colores que utilizan ellos. La diferencia de colores empleados radica, más bien, en el tipo de figura que hay que colorear, dependiendo de si ésta es de un sexo o del otro.

\section{Dibujo de las estrellas (H5)}

Los resultados demostraron que los colores más utilizados por las niñas para pintar las tres estrellas fueron: el amarillo, el rosa y el violeta. Y los colores que más utilizaron ellos fueron el amarillo, el rojo y el azul claro.

\section{Color favorito (H6a y H6b)}

Un alto porcentaje de las niñas $(40,9 \%)$ indicó como su color favorito el rosa, seguido del violeta $(27,3 \%)$. El resto de colores fueron votados con porcentajes menores o nulos. Por su parte, los niños eligieron el rojo como su color favorito $(39,3 \%)$, seguido muy de cerca por el azul claro $(32,1 \%)$.

\section{Diferencias en el uso de los colores según los grupos de edad (H7)}

En relación a las diferencias de colores empleados para pintar cada figura sexuada por parte de alumnado de 4-5 años y alumnado de 6-7 años, los altos porcentajes se dan a la hora de pintar de rosa o violeta la figura de la niña, que es cuando ambos grupos de edad coinciden. En los resultados también se observa una pequeña diferencia: el grupo de 4-5 años tiende a utilizar más variedad, recurriendo a todos los colores para pintar indistintamente si se compara con el grupo de alumnado de 6-7 años, que parece diferenciar ligeramente los colores empleados para pintar las dos figuras sexuadas.

Presencia del color favorito en una de las tres estrellas pintadas (H8)

El $62 \%$ de la muestra que participó en el estudio pintó de su color favorito uno de las tres estrellas presentadas, frente a un $38 \%$ que no lo hizo. 


\section{Discusión}

A partir del análisis de los resultados pudo comprobarse cuáles de las hipótesis se habían cumplido y cuáles no. Se cumplieron las hipótesis $H 1, H 2, H 4$ y H6a, mientras que de las demás una no se cumplió y las otras parcialmente.

No se cumplió la hipótesis $H 6 b$ de que los niños iban a escoger la mayoría el azul como su color favorito, pues el color más seleccionado fue el rojo.

Por otra parte, se cumplieron parcialmente las hipótesis $H 3, H 5, H 7$ y $H 8$. En relación a la variedad de colores utilizados por cada sexo para pintar las figuras de la niña y del niño (H3) no se cumplió totalmente que las y los participantes utilizaban mayor número de colores para pintar la figura que era de su mismo sexo, pues ellos pintaron, prácticamente, con el mismo número de colores ambas figuras. Sí se cumplió la hipótesis para el caso de ellas, que pintaron con más colores la figura de la niña. En cuanto al dibujo de las tres estrellas (H5) las niñas y los niños solo coincidieron en el uso del amarillo para colorear las estrellas. Parcialmente también se cumplió la hipótesis $H 7$, ya que se observaron unas pequeñas diferencias en el uso de los colores por parte del alumnado de 4-5 años y el de 6-7 años para pintar las dos figuras sexuadas. Y de la hipótesis $H 8$ no se puede decir tampoco que se cumplió totalmente, pues dados los porcentajes obtenidos no toda la muestra participante pintó una de las estrellas de su color favorito, sino que más bien se cumplió en el $62 \%$ de la muestra.

Una vez discutidos los resultados, cabe mencionar que la principal limitación del estudio fue que la muestra de población era bastante pequeña. No obstante, no se descarta la idea de ampliarla en un futuro.

Y como reflexión final hay que destacar la idea de que los colores no representan a un género o al otro, sino que son la cultura y la sociedad quienes los etiquetan de esa forma. Esta idea la tienen que tener muy presente todas las personas que educan desde los diferentes ámbitos de intervención educativa.

\section{Referencias}

Bravo, R. S. (1996). Tesis Doctorales y trabajos de Investigación Científica. Madrid: Editorial Paraninfo. Karniol, R. (2011). The Color of Children's Gender Stereotypes. Sex Roles, 119-132. http://dx.doi.org/10.1007/s11199-011-9989-1

Lameiras, M., Carrera, M. V., \& Rodríguez Castro, Y. (2013). Sexualidad y salud. El estudio de la sexualidad humana desde una perspectiva de género. Servicio de Publicacións da Universidade de Vigo.

Navarro, R., Martínez, V., Yubero, S., \& Larrañaga, E. (2014). Impact of Gender and the Stereotyped Nature of Illustrations on Choice of Color: Replica of the Study by Karniol (2011) in a Spanish Sample. Gend. Issues, 142-162. http://dx.doi.org/10.1007/s12147014-9122-1

Pizarro, S. G.-B. (1978). El color en el arte infantil. Madrid.
Santillana. (2009). Juegos divertidos para el cole. Mi primera casa del saber. Madrid. 